\title{
Porous Modelling of Cardiac Perfusion under Contraction to Demonstrate the Distribution of Therapeutic Nanoparticles
}

\author{
Alexandra K Diem ${ }^{1}$, Kristian Valen-Sendstad ${ }^{1}$ \\ ${ }^{1}$ Simula Research Laboratory, Fornebu, Norway
}

\begin{abstract}
Delivery of therapy-loaded nanoparticles (NP) via inhalation is an innovative technology shown to successfully reduce heart failure in mice, improving the efficiency of drug delivery, and reducing adverse side effects. However, how exactly therapeutic NP are distributed and absorbed by heart tissue remains poorly understood, and accelerating this technology to humans is a major challenge. Working towards overcoming this problem we developed an open-source finite element model of myocardial perfusion based on porous media, where perfused tissue is treated as a sponge-like continuum material, driven by a continuum model of cardiac contraction to simulate perfusion patterns. We use our novel particle-tracking based method that remains numerically stable under high Peclet number flow to enable the study of NP distribution.
\end{abstract}

\section{Introduction}

Improving the efficiency of drug delivery to increase efficacy and reduce adverse side effects has been a major goal for medical innovation for decades. In particular, state-of-the-art therapy for cardiac diseases such as heart failure (HF) following adverse remodelling involves invasive surgical procedures and broad-spectrum drugs that can carry as much or more risk for the patient than the disease itself. A novel approach to overcome this problem is the targeted delivery of safe, therapy-loaded nanoparticles (NP) via inhalation. This innovative medical technology has successfully been shown to restore cardiac function, and thereby reduce HF, in a mouse model with diabetic cardiomyopathy [1], and is thus a promising candidate to revolutionise cardiac therapy. However, our understanding of how exactly therapeutic NP are distributed within the heart tissue remains limited. Resolving the mechanisms and patterns of perfusion, their relation to HF, and effects on NP distribution is a major priority to accelerate the development of this innovation, and efficient computational modelling has the potential address some of these questions.
Here, we present an open-source finite element model of myocardial perfusion based on porous mechanics, coupled with our recently developed particle-tracking based method to study NP distribution [2]. The discrepancy of scales between NP, and smaller and larger arterioles renders conventional advection-diffusion kinetics practically unusable. We demonstrate how this discrepancy can be exploited to track NP or other tracers with a significant reduction of computational cost, allowing for more efficient multiphysics simulations.

\section{Methods}

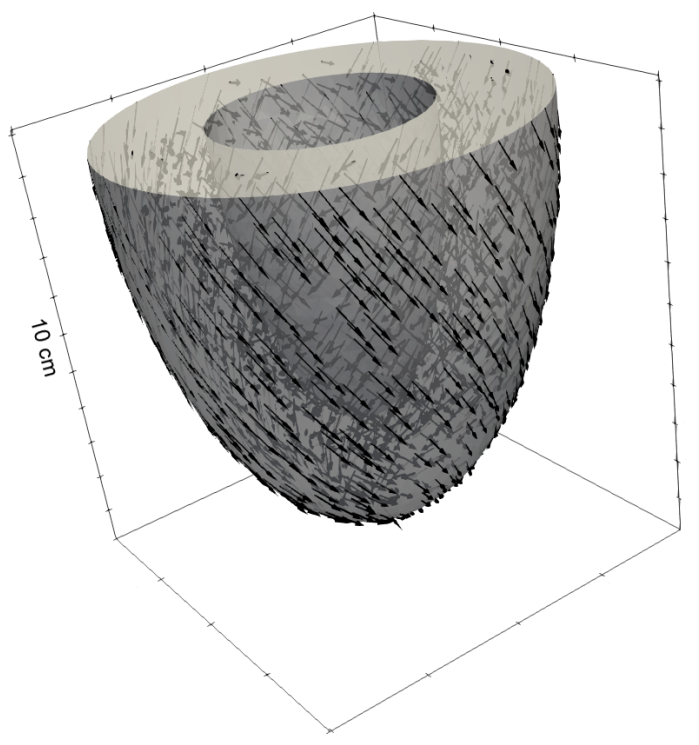

Figure 1. Geometry of the idealised ellipsoid left ventricle geometry used in our simulations. The arrows indicate fiber orientations used to calculate permeability tensors.

We use the finite element method to model left ventricle perfusion under cardiac contraction and solve the equations detailed below on an idealised ellipsoid left ventricle geometry (Figure 1), using the parameters listed in Table 1. 


\subsection{Porous model of cardiac perfusion}

Table 1. Simulation parameters.

\begin{tabular}{lcr}
\hline \hline Permeability & comp. 1 & $1 \mathrm{~mm}^{2} \mathrm{kPa}^{-1} \mathrm{~s}^{-1}$ \\
& comp. 2 & $10 \mathrm{~mm}^{2} \mathrm{kPa}^{-1} \mathrm{~s}^{-1}$ \\
& comp. 3 & $20 \mathrm{~mm}^{2} \mathrm{kPa}^{-1} \mathrm{~s}^{-1}$ \\
\hline Porosity & comp. 1 & 0.021 \\
& comp. 2 & 0.029 \\
& comp. 3 & 0.061 \\
\hline Eff. diffusion & comp. 3 & $1 \mathrm{~mm}^{2} \mathrm{~s}^{-1}$ \\
\hline Coupling coeff. & $\beta_{1 \rightarrow 2}$ & $0.02 \mathrm{~Pa}^{-1} \mathrm{~s}^{-1}$ \\
& $\beta_{2 \rightarrow 3}$ & $0.05 \mathrm{~Pa}^{-1} \mathrm{~s}^{-1}$ \\
& $\gamma_{1 \rightarrow 2}$ & 0.1 \\
& $\gamma_{2 \rightarrow 3}$ & 0.1 \\
\hline \hline
\end{tabular}

The heart is perfused by a dense network of arterioles and capillaries whose diameters vary between ten and hundreds of $\mu \mathrm{m}$ that are impossible to image directly [3]. Thus, we approximate this dense network of blood vessels within myocardial tissue as a $N$-compartment porous continuum medium using Darcy's law [4]:

$$
-\nabla \cdot\left(\boldsymbol{K}_{i} \cdot \nabla p_{i}\right)+\sum_{k=1}^{N} \beta_{i \rightarrow k}\left(p_{i}-p_{k}\right)=s_{i} \quad \text { in } \Omega,
$$

where $i$ represents a distinct scale of arteriole radius with its own pressure distribution $p_{i}$, and permeability tensor $\boldsymbol{K}_{i}$. Whilst the exact geometry of the blood vessels perfusing the left ventricle is not possible, the permeability tensor allows for the incorporation of the macroscale geometry of the flow by following myocardial fiber directions. Fiber directions have been calculated using a Laplace-Dirichlet rule-based method [5] (Figure 1). Flow across compartments is governed by the pressure difference, scaled by a compartment coupling coefficient $\beta_{i \rightarrow k}$. Here, we follow the approach by Michler et. al. [4] to divide the porous model into three compartments to represent large (compartment 0 ), and small arterioles (compartment 1), as well as capillaries (compartment 2). In the following equations, subscripts refer to the numerical compartment labels.

Blood supply is modelled as a Dirichlet boundary condition that is uniformly applied on the epicardium

$$
p_{0}=p_{\text {coronary }} \quad \text { on } \Gamma_{\text {epi }} \text {. }
$$

Outflow is modelled via a uniform sink term that depends on the capillary pressure and represents venous outflow

$$
s_{2}=-\frac{0.01}{\mathrm{Pas}}\left(p_{2}-2933 \mathrm{~Pa}\right),
$$

where $2933 \mathrm{~Pa}=22 \mathrm{mmHg}$ represents venous pressure [6].
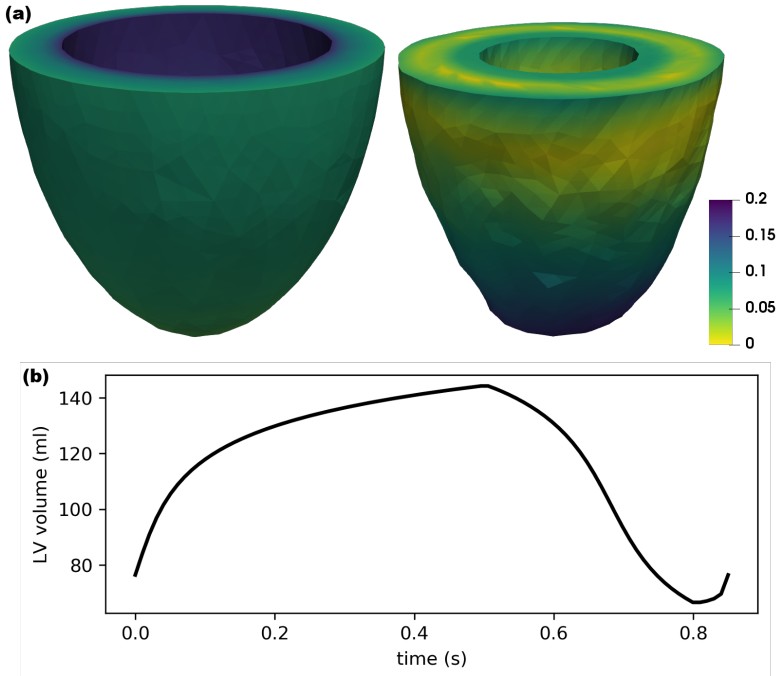

Figure 2. Left ventricle displacement. (a) Maximum expansion at end diastole (left), and contraction at peak systole (right) during one cardiac cycle, scaled by a factor of two. (b) Left ventricular volume loop.

Velocity is evaluated using

$$
\boldsymbol{w}_{i}=-\Phi_{i} K_{i} \nabla p_{i} \quad \text { in } \Omega
$$

with porosities $\Phi_{i}$ and zero Neumann boundary conditions.

Perfusion pressure is driven by cardiac contraction by solving the momentum balance equation

$$
\nabla \cdot(F S)=0
$$

for a Holzapfel-Ogden material model [7] subject to the constitutive law

$$
\begin{aligned}
\Psi=\frac{a}{2 b} \exp \left[b\left(I_{1}-3\right)\right] \\
+\frac{a_{f}}{2 b_{f}}\left[\exp \left[b_{f}\left(I_{4 f_{0}}-1\right)^{2}\right]-1\right] \\
+\frac{a_{s}}{2 b_{s}}\left[\exp \left[b_{s}\left(I_{4 s_{0}}-1\right)^{2}\right]-1\right] \\
\quad+\frac{a_{f s}}{2 b_{f s}}\left[\exp \left(b_{f s} I_{8 f_{0} s_{0}}^{2}\right)-1\right] .
\end{aligned}
$$

with material constants $a, b$, and invariants $I$ of the right Cauchy-Green deformation tensor $\boldsymbol{C}$, where subscript $f$ refers to values in the fiber direction, and subscript $s$ refers to values in the sheet direction. The mechanics model is solved using the cardiac mechanics solver pulse [8]. Figure 2 shows displacements of the left ventricle that was calculated from the model and used as the driving force for the perfusion model. 


\subsection{Nanoparticle tracking for high Peclet numbers}

NP distribution is modelled using our particle-tracking based method, which is described in detail in [2]. The discrepancy in size between therapeutic NP and blood flow leads to high Peclet numbers (Pe), which induce numerical errors when solving conventional advection-diffusion (AD) kinetics using finite elements. To overcome this issue, we separate the problem into two parts:

- advection in large and small arterioles, and

- diffusion in capillaries.

A bolus of NP is advected using a first-order transient model, where position $\boldsymbol{x}$ evolves according to $\boldsymbol{w}$. In the capillaries we approximate blood flow via an effective diffusion coefficient.

The full model thus reads

$$
\begin{gathered}
\frac{\partial \boldsymbol{x}_{0,1}}{\partial t}=\boldsymbol{w}_{0,1} \\
\frac{\partial c_{2}}{\partial t}=\nabla \cdot\left(D_{2} \nabla c_{2}\right) .
\end{gathered}
$$

\section{Results}

\subsection{Myocardial perfusion pressure}

Myocardial perfusion pressure and angular velocity are shown in Figure 3 (top and bottom row, respectively). In the large arterioles (compartment 0 ) is largely dominated by stress originating from tissue contraction with a range that is much larger than predicted by pure porous models $[2,4]$. The narrow pressure ranges in small arterioles and capillaries suggest that coupling coefficients $\beta$ are sensitive to the pressure range range within the large arterioles, and thus differences across compartments. Velocities are given in the angular direction and are displayed on a logarithmic scale.

\subsection{Nanoparticle tracking}

NP distribution is displayed in Figure 4 as a fraction of full perfusion. NP are injected at 5 locations around the heart are not consumed by the tissue to allow for the study of distribution patterns. After three cardiac cycles $(t=$ $2.5 \mathrm{~s}$ ) the heart has reached a minimum of $10 \%$ perfusion, while locations around injection sites reach $50 \%-90 \%$.

\section{Discussion and Conclusion}

We have successfully developed an efficient and numerically stable tracking method for NP or tracers independet of Pe with applicability to organ perfusion in general. Here, we have used this method to illustrate perfusion and distribution of tracers in an idealised left ventricle geometry of a human heart. This work presents a first model of a forward coupling of cardiac mechanics to a porous perfusion model with tracer tracking.

The discrepancies in perfusion pressure between our mechanics driven model and pure perfusion models indicates the sensitivity of parameters such as permeability and especially inter-compartment coupling coefficients to the mechanics. A large-scale sensitivity analysis is thus required to resolve the mechanisms behind organ perfusion and cardiac diseases that develop over large timeframes, such as heart failure due to myocardial remodelling. It should be mentioned that while the choice of $N=3 \mathrm{com}$ partments can be justified by its ability to assign the labels large, and small arterioles, and capillaries, this choice is essentially arbitrary. It is thus interesting to investigate the effects of representing the vasculature by more or fewer compartments.

Furthermore, this study sets out the framework for NP drug delivery optimisation simulations to aid the development of targeted treatment for cardiovascular diseases. This is achieved by the efficiency of the particle tracking method that allows for the solution of multiple cardiac cycles on a conventional laptop within minutes. A fully twoway coupled poroelastic model that additionally takes into account the influence of perfusion fluid contents onto the mechanical constitutive law is in development.

\section{Acknowledgments}

This work was supported by The European Research Council through a Horizon 2020 (grant no. 720834), carried out at the Department of Computational Physiology at Simula Research Laboratory.

\section{References}

[1] Miragoli M, et al. Inhalation of peptide-loaded nanoparticles improves heart failure. Sci Transl Med 2018; 10(424):eaan6205.

[2] Diem A, Valen-Sendstad K. Timelapsing perfusion: Proof of concept of a novel method to study drug delivery in whole organs. Biophys J 2019; accepted.

[3] Ghekiere $\mathrm{O}$, et al. Image quality in coronary ct angiography: Challenges and technical solutions. Brit J Radiol 2017; 90(1072):20160567.

[4] Michler C, et al. A computationally efficient framework for the simulation of cardiac perfusion using a multicompartment darcy porous-media flow model. Int J Numer Meth Bio 2013;29:217-232.

[5] Bayer J, et al. A novel rule-based algorithm for assigning myocardial fiber orientation to computational heart models. Ann Biomed Eng 2012;40(10):2243-54.

[6] Chilian W. Microvascular pressures and resistances in the 

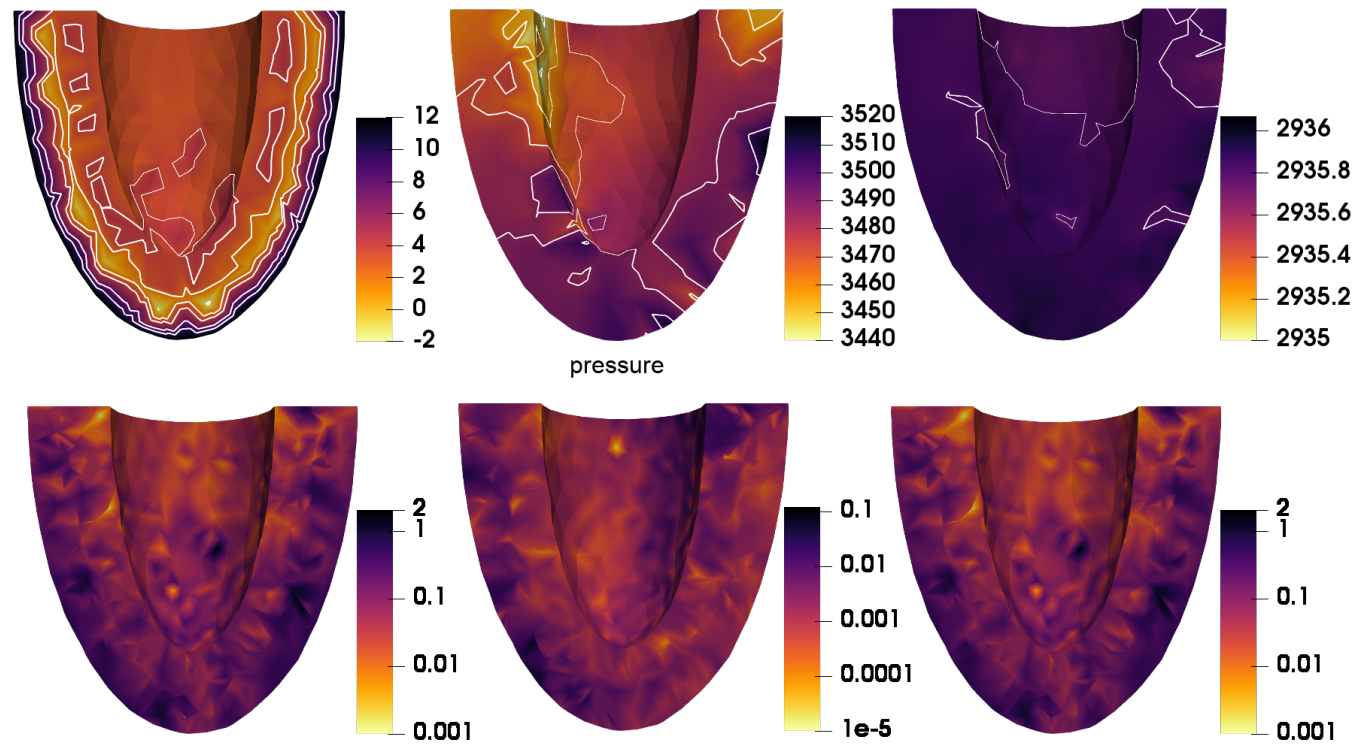

pressure

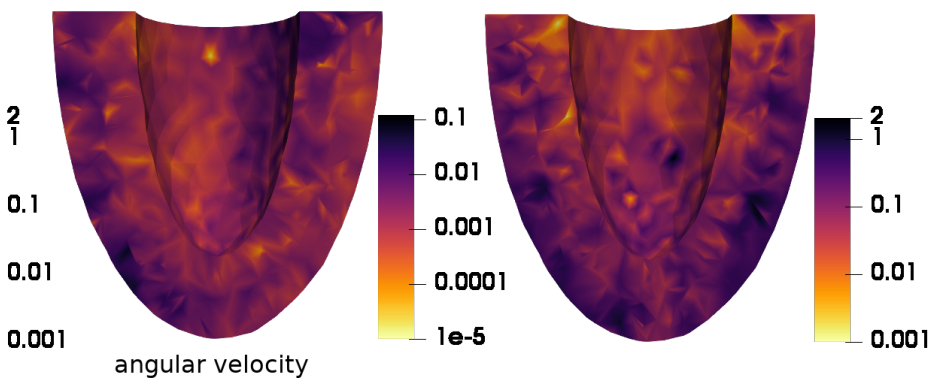

Figure 3. Myocardial perfusion pressure in the left ventricle (top row, units $\mathrm{kPa}$ for compartmet 0 and units $\mathrm{Pa}$ for compartments 1 and two), and radial velocity (bottom row, units $\mathrm{mm} \mathrm{s}^{-1}$ ).
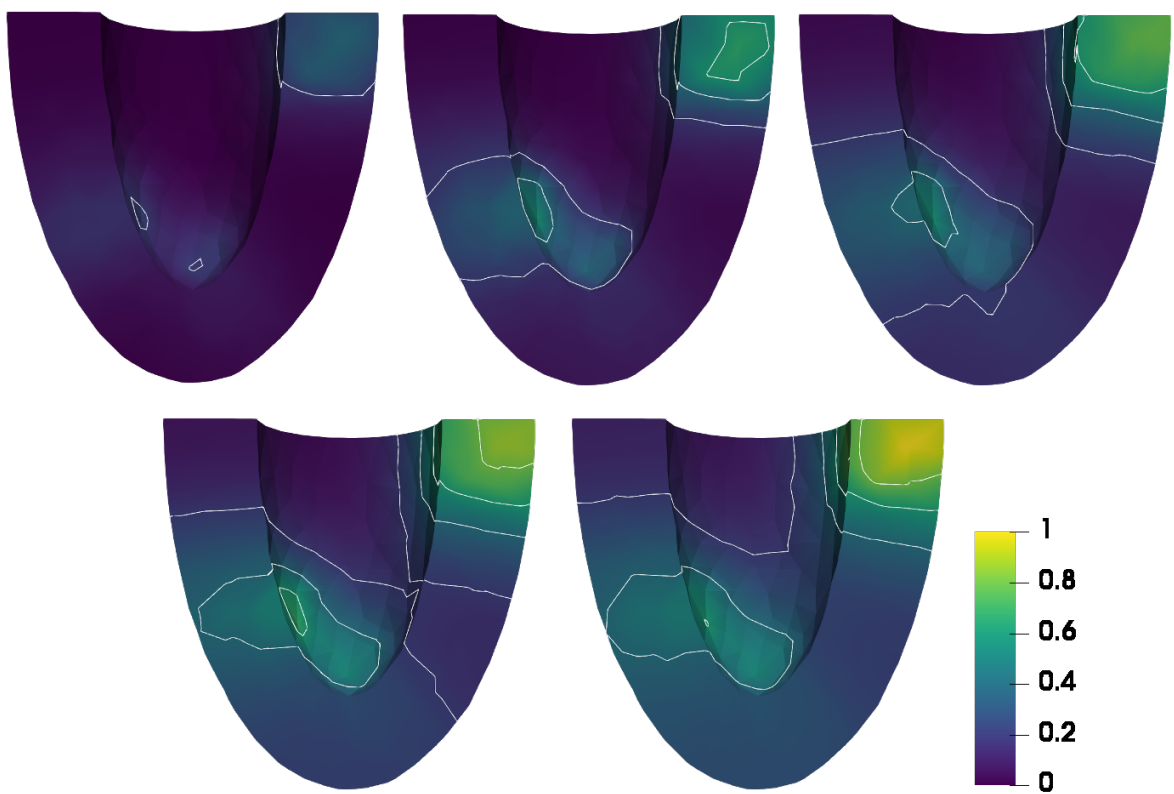

Figure 4. NP distribution as a fraction of full perfusion at $t=0.5 \mathrm{~s}, 1.0 \mathrm{~s}, 1.5 \mathrm{~s}, 2.0 \mathrm{~s}, 2.5 \mathrm{~s}$.

left ventricular subepicardium and subendocardium. Circ Res 1991;69(3):561-70.

[7] Holzapfel G, Ogden R. Constitutive modelling of passive myocardium: A structurally based framework for material characterization. Philos Trans Royal Soc A 2009; 367(1902):3445-75.

[8] Finsberg H. pulse: A python package based on fenics for solving problems in cardiac mechanics. JOSS 2019; 4(41): 1539 .
Address for correspondence:

Alexandra K Diem

Simula Research Laboratory, Fornebu, Norway alexandra@simula.no 